\title{
Penfigoide de las mucosas relacionado con probable enfermedad inflamatoria intestinal: a propósito de un caso
}

\section{Mucous membrane pemphigoid associated to a probable inflammatory bowel disease: a case report}

\author{
Yasmin Alvaro ${ }^{1}$, Liliana Olivares ${ }^{1}$, Olga Forero ${ }^{1}$, María E. Candiz ${ }^{1}$ y Esteban Maronna ${ }^{2}$ \\ ${ }^{1}$ Departamento de dermatología; '2Departamento de dermatopatología. Hospital de infecciosas F.J Muñiz, Buenos Aires, Argentina
}

\section{Resumen}

El penfigoide de las mucosas, antes llamado penfigoide cicatrizal, es una enfermedad ampollar autoinmunitaria poco frecuente, que se caracteriza por ampollas tensas y erosiones, que comprometen sobre todo a las mucosas y al resolverse dejan cicatrices que pueden producir disfunciones notorias. Se presenta el caso de un paciente con penfigoide de las mucosas grave y extendido, con afectación cutánea y una especial relación con una probable enfermedad inflamatoria intestinal. Se realiza una revisión de casos notificados en las publicaciones médicas con esta relación.

Palabras clave: Penfigoide cicatrizal. Penfigoide de las mucosas. Enfermedad inflamatoria intestinal.

\begin{abstract}
Mucous membrane pemphigoid, previously known as cicatricial pemphigoid, is a rare autoimmune bullous disease that affects primarily mucous membranes and occasionally the skin. It is characterized by tense blisters, erosions and subsequent scarring that can cause severe impairment. We present a patient with mucous membrane pemphigoid and cutaneous involvement, highlighting its association with an inflammatory bowel disease. We reviewed the literature in search for other cases with this association.
\end{abstract}

Key words: Cicatricial pemphigoid. Mucous membrane pemphigoid. Inflammatory bowel disease.

\section{Introducción}

El penfigoide de las mucosas (PM), antes denominado penfigoide cicatrizal, es una enfermedad ampollar autoinmunitaria dermoepidérmica, crónica, poco frecuente, que se distingue por ampollas tensas y erosiones que dejan cicatrices al resolverse ${ }^{1}$. Afecta en particular a las mucosas, las más frecuentes de las cuales son la oral y la ocular, con disfunciones graves que producen una gran morbimortalidad relacionada con las sinequias secuelares².

Existen escasas publicaciones que vinculen al PM con la enfermedad inflamatoria intestinal (EII). Se presenta el caso de un paciente con PM grave y compromiso extenso, tal vez en relación con Ell.
Correspondencia:

*Yasmín Álvaro

E-mail: yasalvaro@ hotmail.com
Disponible en internet: 06-10-2021 Med Cutan Iber Lat Am. 2021:49(Suppl 1):30-35 www.MedicinaCutanealLA.com 0210-5187/C 2021 Colegio Ibero Latinoamericano de Dermatología A.C. (CILAD). Publicado por Permanyer. Este es un artículo open access bajo la licencia CC BY-NC-ND (http://creativecommons.org/licenses/by-nc-nd/4.0/). 


\section{Caso clínico}

Paciente masculino de 57 años que consultó por dermatosis en el polo cefálico y tronco de cinco meses de evolución, en nexo con disfagia, pérdida de peso de $10 \mathrm{~kg}$ y disminución de la agudeza visual. Tenía además el antecedente de diarrea crónica, fisuras anales y absceso perineal que precedían cuatro meses a la dermatosis.

Exploración física: sinequia conjuntival y ectropión en el ojo derecho con inyección conjuntival bilateral (Fig. 1). En la cavidad oral mostraba numerosas erosiones de fondo rojizo y sangrante en paladar y mucosa gingival (Fig. 2); ampolla tensa en el pilar anterior de la fosa amigdalina. En la cara había cicatrices atróficas y erosiones (Fig. 3). Lesiones de similares características se repetían en el tronco superior (Fig. 4), pliegues inguinales y glande (Fig. 5). En la región anal: fisuras dolorosas. Signo de Nikolsky negativo.

Estudios complementarios: a) estudios de laboratorio completos sin particularidades; b) serologías para HIV, hepatitis B y C negativas; C) anticuerpos anti-Saccharomyces cerevisiae (ASCA), IgA de $100 \mathrm{UI} / \mathrm{L}$ (VN hasta 20UI/L)-lgG de $60 \mathrm{UI} / \mathrm{L}$ (VN hasta $20 \mathrm{UI} / \mathrm{L}$ ); d) biopsia de piel de dorso para histopatología: ampolla subepidérmica con moderado infiltrado inflamatorio constituido por linfocitos y abundantes eosinófilos (Figs. 6 y 7); e) inmunofluorescencia directa (IFD) con técnica de salt split: positiva para anticuerpos IgA con depósito en zona de la membrana basal (ZMB), costado epidérmico; f) inmunofluorescencia indirecta (IFI): positiva para anticuerpos IgG en la ZMB (título: 1/40); g) serologías por estudio de inmunoabsorción ligado a enzima (ELISA) para desmogleínas 1 y 3, el antígeno menor del penfigoide ampollar (BP180 o BPAG2) (dominio NC16A), el antígeno mayor del penfigoide ampollar (BP230 o BPAG1) (dominio carboxiterminal-C terminal), envoplaquina y colágeno VII (dominio no colágeno tipo 1, NC1): negativas.

Para valorar el compromiso de mucosas se realizaron: estudio oftalmológico (anquilobléfaron en ojo derecho y conjuntivitis crónica bilateral); laringoscopia (erosiones, con biopsia inespecífica); videoendoscopia digestiva alta y tránsito esofágico (erosiones en paladar y faringe con estenosis esofágica no franqueable); anoscopia (fisuras anales y atrofia de mucosa anal); biopsias de mucosa esofágica y anal (ampolla subepidérmica con infiltrado con linfocitos, algunos eosinófilos y neutrófilos [Fig. 8); resonancia magnética nuclear de piso pélvico: absceso perineal.



Figura 1. Afectación en el ojo derecho con sinequia conjuntival y ectropión.

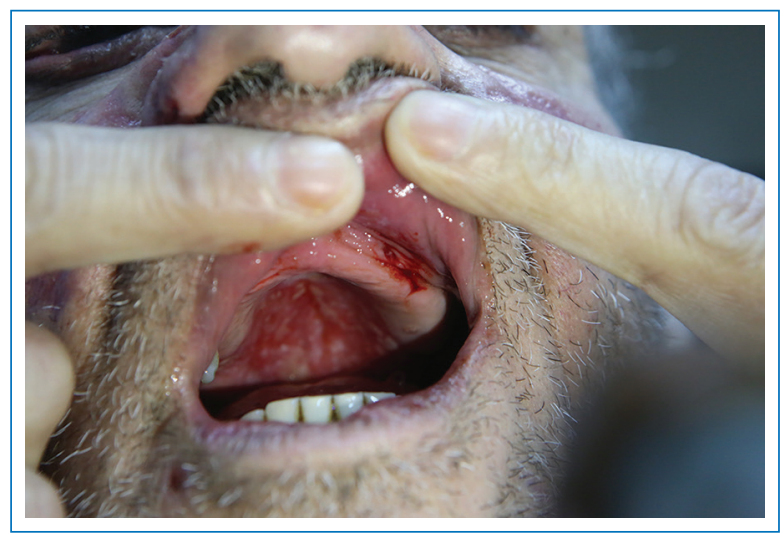

Figura 2. Compromiso oral: erosiones en el paladar y la mucosa gingival.

Con los resultados obtenidos se estableció el diagnóstico de PM (ocular, oral, laríngea, esofágica, genital, anal y cutánea) en relación con probable Ell en estudio.

Se indicó tratamiento con dexametasona (24 mg/día IV) junto con azatioprina (2 mg/kg/día VO) y gotas oftálmicas con ciclosporina y eritromicina. Un mes después, por falta de mejoría, se inició gammaglobulina intravenosa (2 $\mathrm{g} / \mathrm{kg}$ dosis total) administrada en cinco días. Al tercer día de la infusión sobrevino sepsis por Staphylococcus aureus resistente a la meticilina y se lo trasladó a la unidad de cuidados intensivos, con choque séptico y muerte posterior.

Si bien el estudio de la Ell no pudo completarse, la diarrea anterior a la dermatosis, las fístulas perianales, el absceso perineal y los títulos elevados de ASCA determinaban una sólida sospecha de Ell. 


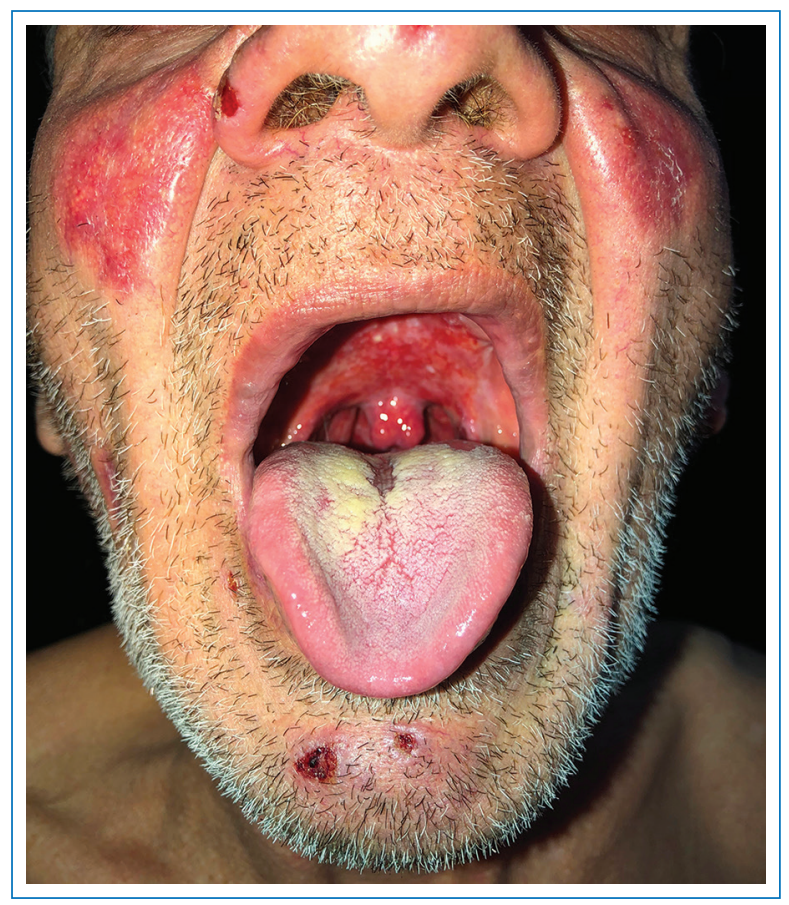

Figura 3. Ampolla tensa en el pilar anterior de la fosa amigdalina y compromiso cutáneo en la cara, con cicatrices atróficas y erosiones.

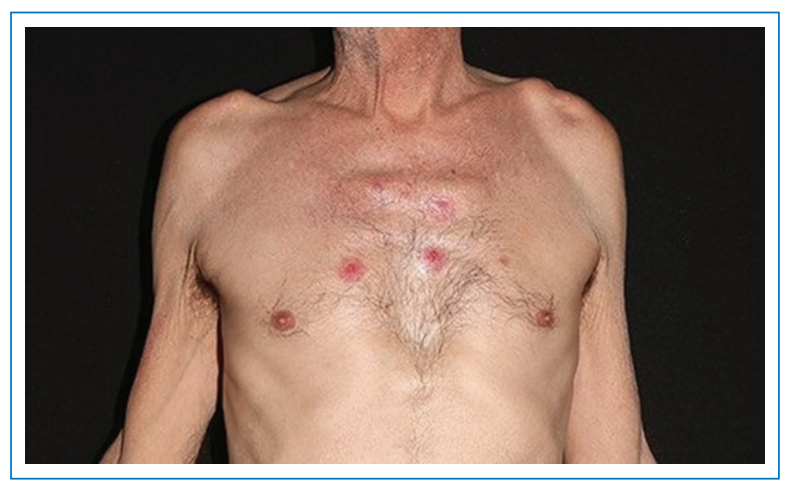

Figura 4. Compromiso cutáneo en el tronco con cicatrices atróficas y erosiones.

\section{Discusión}

El PM es una enfermedad ampollar autoinmunitaria dermoepidérmica, crónica, infrecuente, que afecta a adultos mayores de 60 años con predominio en el sexo femenino ${ }^{1-3}$.

Con respecto a la patogenia, si bien no se conoce con exactitud, se ha propuesto que existen diferentes factores. Por un lado, se relaciona con una predisposición genética determinada por el alelo HLA$\mathrm{DQB1}{ }^{*} 0301^{3}$. Además, es posible que exista algún

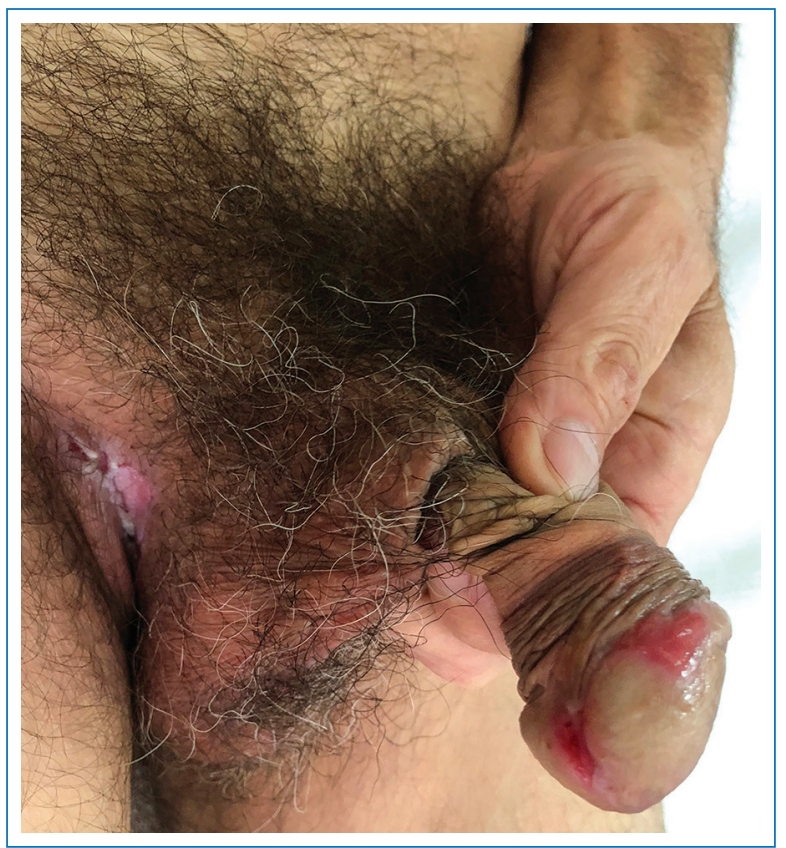

Figura 5. Compromiso genital: erosiones en el glande.



Figura 6. Histopatología de la biopsia del dorso: ampolla subepidérmica con leve infiltrado inflamatorio perivascular en la dermis superficial (H-E, 100x).

factor aún no determinado, quizá virus, fármacos o afecciones preexistentes, que precipitarían una respuesta inmunitaria o inflamatoria con exposición de antígenos previamente ocultos y formación de anticuerpos contra componentes de la membrana basal (fenómeno de dispersión de epítopos o epitope spreading) ${ }^{3}$. Se ha demostrado que es una entidad en la que los anticuerpos circulantes pueden afectar a uno o más antígenos, lo que se traduce en los diferentes resultados en la inmunofluorescencia con salt split, como se 


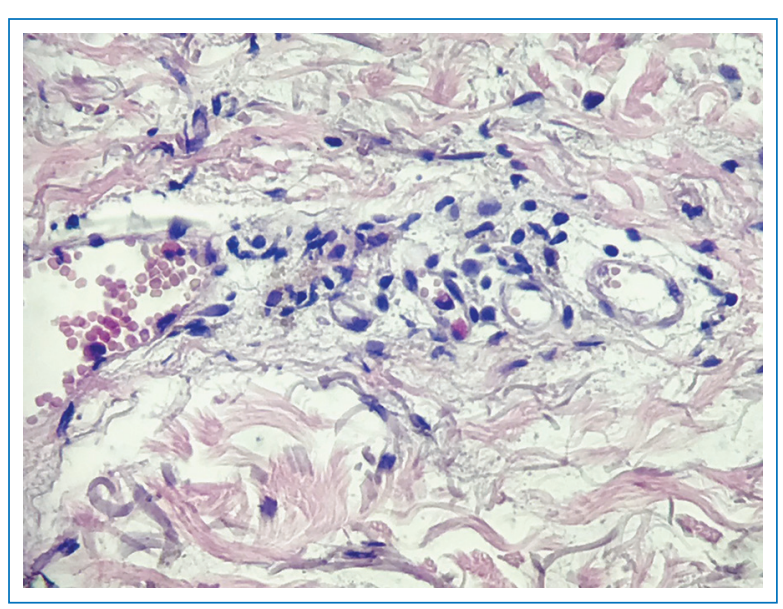

Figura 7. Histopatología de la biopsia del infiltrado inflamatorio constituido por linfocitos y algunos eosinófilos del dorso (H-E, 400x).

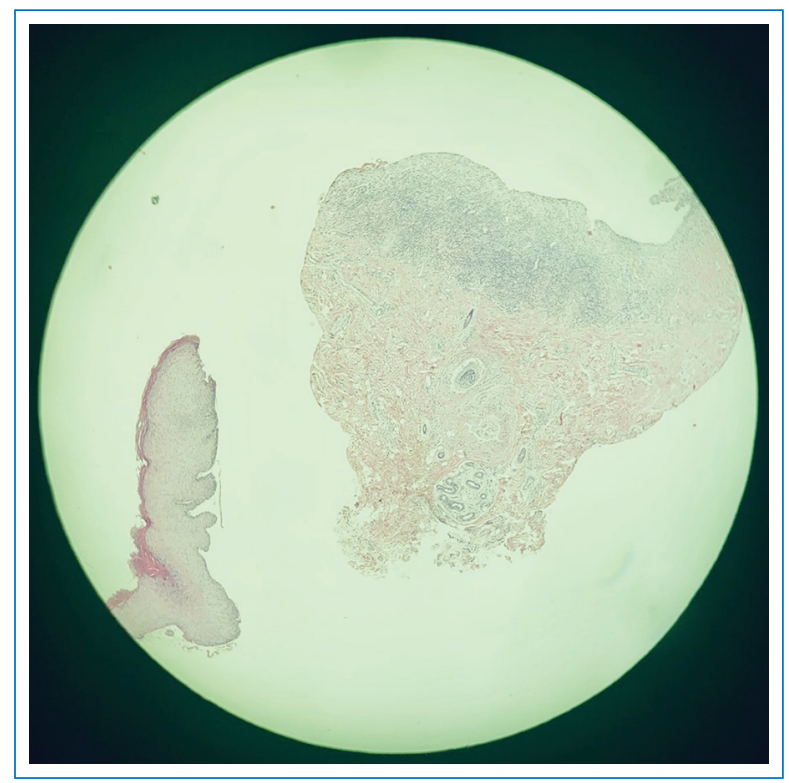

Figura 8. Biopsia de la mucosa perianal: ampolla subepidérmica $(H-E, 40 x)$.

describe más adelante. Dichos antígenos son BP180, laminina 332 (laminina 5), subunidades $\beta 4$ y $\alpha 6$ de la integrina $y$, con menor frecuencia, BP230 y colágeno de tipo $\mathrm{VII}^{4}$. El extremo $\mathrm{C}$ terminal de BP180 y la laminina 332 son los principales antígenos del $\mathrm{PM}^{5}$.

El compromiso de la mucosa oral es el más frecuente y en muchas ocasiones la gingivitis descamativa representa la primera y única manifestación`. La afectación ocular es la segunda en frecuencia; inicia como una conjuntivitis crónica bilateral, evoluciona con tendencia a la formación de adherencias de la mucosa conjuntival (simbléfaron) y obliteración del saco conjuntival (anquilobléfaron) que pueden conducir a la ceguera $^{3}$.

Otras mucosas afectadas en orden de frecuencia son nasofaríngea (sinusitis crónica, alteración del flujo aéreo, epistaxis), anogenital (estenosis, tenesmo), laríngea (disfonía, ronquera e incluso obstrucción de las vías respiratorias) y esofágica (disfagia, estenosis) ${ }^{3,6}$. En el $25 \%$ de los casos se observa además compromiso cutáneo, que se manifiesta en la forma de ampollas tensas que generalmente se asientan sobre una base eritematosa y se resuelven con cicatrices atróficas'.

Con respecto al diagnóstico, se utilizan en clínica cinco métodos: IFD, IFI, histopatología, serologías por ELISA y electrotransferencia. La IFD es positiva en $80 \%$ a $100 \%$ de los casos y se observa un depósito lineal de $\lg G$ o $C 3$ y de modo ocasional IgA en la ZMB ${ }^{4}$.

La IFI identifica anticuerpos circulantes $\lg G \circ \lg A$ en el suero del paciente, pero es positiva sólo en $20 \%$ a $30 \%$ de los $\operatorname{casos}^{6}$. Con la técnica de salt split se observa que las inmunoglobulinas se fijan con mayor frecuencia en el lado epidérmico de la piel, aunque algunas veces pueden hacerlo a nivel dérmico o en ambos lados de acuerdo con el antígeno causal ${ }^{4}$.

Con respecto a la histopatología, se reconocen ampollas subepidérmicas con infiltrados inflamatorios constituidos por linfocitos, histiocitos y también cantidades variables de neutrófilos y eosinófilos'.

Las serologías por ELISA disponibles en el servicio de los autores permiten en la actualidad la detección de anticuerpos en el suero del paciente contra los epítopos más frecuentes de los seis antígenos principales de las enfermedades ampollares autoinmunitarias (desmogleínas 1 y 3 , envoplaquina, dominio NC16A del BP180, C-terminal del BP230 y sector NC1 del colágeno tipo VII) ${ }^{7}$. En los casos de PM, en los que los antígenos son los epítopos mencionados de BP180, BP230 y colágeno de tipo VII, las serologías por ELISA pueden ser una herramienta útil. Sin embargo, estos antígenos pueden tener falsos negativos si el anticuerpo se dirige a un epítopo o antígenos no presentes en este kit serológico (laminina 332, subunidades $\beta 4$ y $\alpha 6$ de la integrina). Además, sólo detecta anticuerpos de tipo IgG, y no IgA, que están presentes en casi $60 \%$ de los pacientes con $\mathrm{PM}^{8}$. Por lo tanto, los resultados positivos contribuyen al diagnóstico, pero los resultados negativos no lo descartan.

El tratamiento depende del sitio afectado, la gravedad de la enfermedad y su avance ${ }^{3,9}$. La mayoría de los 
autores considera que los pacientes se pueden dividir en los de bajo riesgo, cuando sólo está afectada la mucosa oral, con o sin compromiso cutáneo, y los de alto riesgo, cuando hay lesiones oculares, faríngeas, laríngeas, esofágicas o genitales ${ }^{9}$. En pacientes de bajo riesgo se utilizan medidas terapéuticas locales, como corticoides tópicos de alta potencia dos a cuatro veces al día o intralesionales e inmunomoduladores como tacrolimus al $0.1 \% 3,4,9$. Se deben acompañar de medidas generales como una estricta higiene bucal y el uso de colutorios con un esquema de gargarismos ${ }^{1}$.

En caso de que no haya respuesta al tratamiento local, se sugiere administrar dapsona a dosis de 50 a $200 \mathrm{mg} /$ día como monoterapia o junto con meprednisona $(0.5-1 \mathrm{mg} / \mathrm{kg} / \mathrm{día})^{9,10}$. En los pacientes de alto riesgo está indicada la combinación de esteroides sistémicos y un inmunosupresor adicional ${ }^{10}$. Entre éstos se recomiendan la azatioprina (2-3 mg/kg/día), ciclofosfamida (1-2 $\mathrm{mg} / \mathrm{kg} / \mathrm{día})$ o micofenolato de mofetilo (30-40 mg/kg/día) junto con meprednisona (1 mg/kg/día) o dosis equivalentes. Se ha descripto el uso de inmunoglobulina intravenosa, como se utilizó en el caso grave y resistente de este paciente, y de agentes biológicos como rituximab, infliximab o etanercept ${ }^{1,11}$. De todas formas, los pacientes con PM requieren un seguimiento de largo plazo por la posibilidad de recurrencia de esta enfermedad crónica ${ }^{1}$.

\section{Penfigoide de las mucosas y su vinculación con enfermerdad inflamatoria intestinal}

Existen publicaciones que vinculan anomalías ampollares autoinmunitarias con la Ell. De ellas, la más citada es la dermatosis por IgA lineal (DIAL) en relación con colitis ulcerosa $(\mathrm{CU})^{12}$. Sin embargo, en las publicaciones médicas se describen casos de otros trastornos ampollares autoinmunitarios como el penfigoide ampollar (PA) y epidermólisis ampollar adquirida (EAA) variedad inflamatoria, pénfigo por IgA y PM, como se describe en este artículo $0^{12-14}$.

Se formuló como hipótesis que la inflamación de la mucosa intestinal en la Ell podría estimular una respuesta inmunitaria humoral a los antígenos epiteliales del colon, con la posterior reactividad cruzada de los anticuerpos contra los antígenos epiteliales de la piel (fenómeno de epitope spreading) ${ }^{12}$. Esto se ha demostrado en los pacientes con EAA, en la cual los anticuerpos IgG contra el colágeno VII reaccionan también en la mucosa colónica (con técnicas de IFD e IFI) ${ }^{14}$.

Diversos trabajos han demostrado otros antígenos expresados a nivel colónico, que también están presentes en la piel. Dichos antígenos son BP180, desmogleína 1, desmocolina 2 y probablemente desmogleína $3^{13,15}$. En el año 2004, Selby, et al. describieron a un paciente con PA y CU y postularon que la plectina (proteína expresada tanto en la piel como en el intestino con una estructura similar al BP230) era el antígeno que ocasionaba la reacción cruzada ${ }^{16}$.

En la mayoría de los casos, la Ell precede al cuadro cutáneo. Se ha señalado que la actividad inflamatoria colónica se relaciona con la actividad de la dermatosis. Esto se observa con mayor claridad en los pacientes con DIAL y CU sometidos a colectomía total con remisión de ambas entidades ${ }^{17}$.

En cuanto a la relación de PM con Ell, se realizó una búsqueda bibliográfica (PUBMED, RIMA) y se identificaron sólo tres casos, dos de ellos con CU y uno con enfermedad de Crohn (EC). En dos de los casos, la Ell precedió al PM por años, mientras que el paciente con EC lo manifestó de manera concomitante ${ }^{12,13,18}$. En este caso, si bien los síntomas digestivos precedieron a las lesiones cutáneas, el tiempo fue sólo de cuatro meses.

En cuanto a los métodos diagnósticos, en el paciente presentado la IFD con técnica de salt split incidió en el techo de la ampolla, lo que hace suponer que los probables antígenos causales según las publicaciones médicas pueden ser BP180, BP230 o la subunidad $\alpha 6$ - $\beta 4$ de la integrina ${ }^{4}$. No fue factible detectar el antígeno causal por técnicas de ELISA. Los resultados serológicos negativos en este paciente podrían explicarse como un falso negativo del BP180 o BP230 o bien como la ausencia de integrinas en el kit?

Si bien en este caso no pudo completarse el diagnóstico de certeza de Ell por la muerte del paciente, la clínica consistente y los títulos de ASCA elevados en valores significativos son muy indicativos de este diagnóstico.

El interés de la publicación reside en presentar una enfermedad ampollar de muy baja frecuencia, grave y extendida que requiere atención interdisciplinaria para valorar la extensión del compromiso de otras mucosas y sus anomalías adjuntas. Por otro lado, se resalta la relación con la Ell, lo que hace aún más particular este caso. Esta vinculación depende tal vez de la inflamación de la mucosa intestinal que precipitaría una reacción inmunitaria humoral, con la posterior reactividad cruzada contra los antígenos epiteliales de la piel.

\section{Agradecimientos}

Los autores agradecen a los doctores Pablo Muñoz, jefe de unidad del servicio, y Miguel Lumi, médico de 
planta especialista, y también al Servicio de Coloproctología del Hospital Udaondo.

\section{Financiamiento}

No se recibió ningún financiamiento para llevar a cabo este artículo.

\section{Conflicto de intereses}

Los autores no tienen ningún conflicto de intereses.

\section{Responsabilidades éticas}

Protección de personas y animales. Los autores declaran que para esta investigación no se han realizado experimentos en seres humanos ni en animales.

Confidencialidad de los datos. Los autores declaran que han seguido los protocolos de su centro de trabajo sobre la publicación de datos de pacientes.

Derecho a la privacidad y consentimiento informado. Los autores han obtenido el consentimiento informado de los pacientes o sujetos referidos en el artículo. Este documento obra en poder del autor de correspondencia.

\section{Bibliografía}

1. Yancey KB. Penfigoide cicatrizal. En: Fitzpatrick Dermatología en medicina general. Goldsmith LA, Katz SI, Gilchrest BA, Paller AS, Leffell D, Wolff K (eds.). 8va ed. Buenos Aires: Panamericana, 2014:616-622.
. Knudson RM, Kalaaii AN, Bruce AJ. The management of mucous membrane pemphigoid and pemphigus. Dermatol Ther. 2010;23(3):268-280.

3. Tula M. Penfigoide de las mucosas. Dermatol Argent. 2012;18(2):16-23.

4. Pérez DL, Forero OL, Olivares L, Candiz ME. Dermatosis ampollares subepidérmicas neutrofílicas. Dermatol Argent. 2016;22(4):171-182.

5. Kamaguchi M, Iwata $\mathrm{H}$. The diagnosis and blistering mechanisms of mucous membrane pemphigoid. Front Immunol. 2019:10:34.

6. Casnati-Gubern BE, Cuestas M. Penfigoide de membranas mucosas. A propósito de dos casos clínicos. Odontoestomatología. 2010;12(15):13-24

7. Candiz ME, Forero O, Olivares L, Muñoz de Toro M, Criniti J, Lanfranconi $\mathrm{M}$, et al. Diagnóstico serológico de patologías ampollares autoinmunitarias. Dermatol Argent. 2018;24(4):177-184.

8. Schmidt E, Benoit S, Kampik D, Hille K, Geerling G. Wiederherstellung der Sehfähigkeit beim Schleimhautpemphigoid durch Keratoprothese aus dem Tibiaknochen. J Dtsch Dermatol Ges. 2017;15(10):1024-1026. German.

9. Santi CG, Gripp AC, Roselino AM, Mello DS, Gordhilo JO, Marsillac PF, et al. Consensus on the treatment of autoimmune bullous dermatoses: bullous pemphigoid, mucous membrane pemphigoid and epidermolysis bullosa acquisita - Brazilian Society of Dermatology. An Bras Dermatol. 2019:94(2 Suppl 1):33-47.

10. Chan LS, Ahmed AR, Anhalt GJ, Bernauer W, Cooper KD, Elder MJ, et al. The first international consensus on mucous membrane pemphigoid: definition, diagnostic criteria, pathogenic factors, medical treatment, and prognostic indicators. Arch Dermatol. 2002;138(3):370-379

11. Amber KT, Maglie R, Solimani F, Eming R, Hertl M. Targeted therapies for autoimmune bullous diseases: current status. Drugs. 2018;78(15):1527-1548.

12. Shipman AR, Reddy H, Wojnarowska F. Association between the subepidermal autoimmune blistering diseases linear $\lg A$ disease and the pemphigoid group and inflammatory bowel disease: two case reports and literature review. Clin Exp Dermatol. 2012;37(5):461-468.

13. Sotiriou MC, Foo CW, Scholes CT, Zone JJ. Immunobullous disease and ulcerative colitis: a case series of six patients. $\mathrm{Br} J$ Dermatol. 2015; 173(3):792-796

14. Chen M, O'Toole EA, Sanghavi J, Mahmud N, Kelleher D, Weir D, et al. The epidermolysis bullosa acquisita antigen (type VII collagen) is present in human colon and patients with Crohn's disease have autoantibodies to type VII collagen. J Invest Dermato. 2002;118(6):1059-1064.

15. Bruckner AL, Fitzpatrick JE, Hashimoto T, Weston WL, Morelli JG. Atypical lgA/lgG pemphigus involving the skin, oral mucosa, and colon in a child: a novel variant of IgA pemphigus? Pediatr Dermatol. 2005;22(4):321-327.

16. Selby L, De Castro F, De Villiers WJ. The association of bullous pemphigoid and ulcerative colitis. Dig Dis Sci. 2004:49(11-12):1768-1770.

17. Walker SL, Banerjee $P$, Harland CC, Black MM. Remission of linear IgA disease associated with ulcerative colitis following panproclocolectomy. Br J Dermatol. 2000;143(6):1341-1342.

18. Woźniak K, Górkiewicz A, Olszewska M, Schwartz RA, Kowalewski C Cicatricial pemphigoid vegetans. Int J Dermatol. 2007;46(3):299-302. 